\title{
Co-Extracting Opinions from Online Reviews
}

\author{
Beema K S \\ Department of Computer Science and Engineering \\ Mangalam college of Engineering \\ Kottayam,India
}

\author{
Mitha Rachel Jose \\ Department of Computer Science and Engineering \\ Mangalam college of Engineering \\ Kottayam,India
}

\begin{abstract}
Exclusion of opinion targets and words from online reviews is an important and challenging task in opinion mining. The opinion mining is the use of natural language processing, text analysis and computational process to identify and recover the subjective information in source materials. This paper propose a Supervised word alignment model, which identifying the opinion relation. Rather than this paper focused on topical relation, in which to extract the relevant information or features only from a particular online reviews. It is based on feature extraction algorithm to identify the potential features. Finally the items are ranked based on the frequency of positive and negative reviews. Compared to previous methods, our model captures opinion relation and feature extraction more precisely. One of the most advantages that our model obtain better precision because of supervised alignment model. In addition, an opinion relation graph is used to refer the relationship between opinion targets and opinion words.
\end{abstract}

\section{Keywords: Opinion mining, Sentiment Analysis, Topical Relation, Opinion Target, Opinion Words}

\section{INTRODUCTION}

Growth of web 2.0 huge number of user generated data is present on web as blogs, reviews, , comments etc. This data involve user's opinions beliefs, sentiment towards particular product, topic, event, news etc. An opinion mining refers to the use of natural language processing to extract the subjective information from source materials. Opinion mining includes opinion feature which is used to specify an attributes of an entity on which consumers state their views and opinions.

Others opinions can be crucial when it's time to make a judgment or choose among numerous opinions. Sentiment analysis is the computational study of people's emotions. Given a set of documents $D$ that contain or sentiments about an object, opinion mining aims to extract attributes and means of the object that have been commented on in each document $d$ $\in D$ and to evaluate the comments are positive, negative or neutral. This fascinating problem is increasingly important in business and society. It has loads of research challenges but promises approaching helpful to anyone interested in opinion analysis and social media analysis. Humans are objective creatures and opinions are significant Being able to interact with customers, has many advantages for information systems.

Textual information in the world can be broadly classified into two main categories, subjective and objectives. Facts are objective statements about entities and events in the web. It also makes it difficult for the producer of the product to keep track and to supervise customer opinions. For the manufacturer has an additional difficulties because lots of commercial sites may sell the similar product and the manufacturer normally produces many kinds of product. To extract the opinions from online reviews, it is unsatisfactory to obtain the overall sentiment about a particular product. That is an opinion has a positive and negative orientation. For Example: poor battery life "

"A stunning design and a big boost to Core i7 but

At this point an opinion about the laptop consisting positive opinion as "stunning design and big boost to core i7" and negative opinion as "poor battery life"
An opinion target is the object about which users express their opinion typically noun or noun phrase, in the above example design.corei7, and battery are the three opinion target. An opinion word is defined as the words that are used to express the users opinions. In the above example stunning, big boost and poor are the opinion words.

Rather than sentiment analysis and feature extraction proposed method mainly focused on Topical relation. That is extracting the current interest or relevance or pertaining or dealing with matters of current or local interest. This means that

\section{RELATED WORKS}

Lots of studies have paying attention on the task of opinion target and opinion word extraction[1], [2], [5] ,[6]. General textual inspection uses part of speech (POS) information (for example, nouns, adjectives, adverbs, and verbs) as a basic form of word-sense description. Some adjectives are good indicators of emotion and guide feature assortment to categorize the sentiment. Also, selected phrases elected by pre-specified POS patterns, usually including an adjective or adverb, help detect sentiments.

Pang and Lee [8] presented survey on sentiment analysis and opinion mining. So as toward survey they explained opinion oriented information right of entry, challenges, opinion categorization and summarization. Many researchers used machine learning methods for emotion examination [3] [4] [7] that involve guidance of classifier on datasets and use the skilled model for new document classification. Some authors optional another method such as dictionary of word lexicons [6].

Qiu et al. $(2009,2011)$ proposed a Double Propagation technique [5] to explain a domain sentiment lexicon and an view target set iteratively. They exploited direct relations between words to extract opinion targets and belief words iteratively. The main limitation of Qiu's technique is that the patterns based on dependency parsing tree may introduce many noises for the great corpora (Zhang et al. 2010). 
Oppressed syntax information [6] to extract opinion targets, and calculated some syntactic patterns to capture the opinion relations among words. The experimental consequences showed that their technique performed better than that of [5].

\section{SYSTEM ARCHITECTURE}

The opinion mining refers to the use of natural language processing, text analysis and computational linguistics to recognize and take out subjective information in basis resources. Opinion mining is generally useful to reviews and social media for a diversity of applications, ranging from marketing to customer service. As of the customer viewpoint, bearing in mind others opinions before purchasing a product is a common performance extended before the survival of Internet.

Rather than feature classification we focused on Topical Relations. In Topical Relation [1] extract the relevant features only from a particular product from online reviews. We first classify the sentences as opinions or facts and then we will examine only the subjective sentence thus improving performance. Also, we would add a smart crawler component so that all the relevant information from various web pages in a website is automatically crawled and extracted upon providing a URL and certain conditions. We determine the relationship between opinion targets and opinion words. We take all nouns are opinion targets and all adjectives are opinion words. An Opinion relation graph is used to refer the relation between opinion targets and opinion words. To model this process, construct a bipartite graph. A bipartite graph (or bigraph) [1], whose vertices can be divided into two disjoint sets $U$ and $V$ (that is, $U$ and $V$ are each independent sets) such as opinion targets and opinion words that every edge connects a vertex in $U$ to one in $V$.

The opinion mining tasks can be widely categorized based on the level at which it is done with the various levels being namely,
a. The document level
b. The sentence level
c. The feature level.

\section{a. The document level}

At the document level sentiment categorization of documents into positives and negatives. Which is done with the assumption made that each document focuses on a particular object and contains opinion from a single opinion holder.

\section{b. The sentence level}

At the sentence level, recognition of opinionated sentences amongst the reviews is done by classifying data into objective and subjective. Subsequently, sentiment classification of the sentences is done moving each sentence into positive, negative based on Naive bayes classifier.

\section{c. The feature level.}

At the feature level, diversity of tasks that are looked for identifying and extracting features from view. After that determining whether the opinions on the features are positive. At last grouping feature synonyms and producing a featurebased opinion summary of multiple reviews/text.
In Topical Relation extract the relevant features only from a particular product from online reviews. We first classify the sentences as subjective (opinions) or objective (facts) and then we will analyze only the subjective sentences thereby improving performance.. Also, we would add a smart crawler component so that all the relevant information from various web pages in a website is automatically crawled and extracted upon providing a URL and certain conditions.

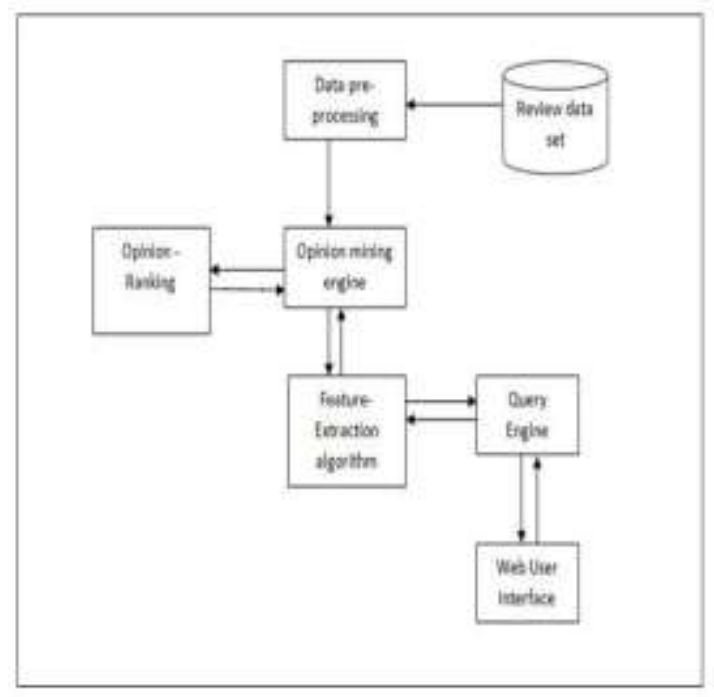

Figure.1 System Architecture

\subsection{The Feature Extraction Algorithm}

The algorithm wished to recognize potential features is called the Feature Extraction algorithm [9]. The idea behind the algorithm is that the nouns for which customers express many number of opinions are most likely to be the important and unique features than those for which users don't state such opinions. This algorithm takes an input is the list of adjectives which are used to express opinions. Pre-processing of words together with removal of stop words. Each and every sentences are parsed using Stanford Parser, then assign a Parts Of Speech (POS) tags to English words based on the context in which they appear.

\section{EXPERIMENTAL EVALUATION}

Consumers can place reviews on web communities, blogs, twitters, product's web site and these comments are called user generated contents. So huge number of data available freely in various websites. We experimented with different reviews on data set in order to measure several parameters of our system. More specifically, we performed three different sets of experiments. In the first line of experiments, we evaluated the performance of our opinion-based feature extraction algorithm, as compared to simple word count algorithms. Naive bayes classifier is used to correctly classifying reviews as positive or negative.

We conducted our experiments using the customer reviews of electronics products such as Iron Box ,Mobile Phone, Trimmers etc. The reviews are collected from the e-commerce site like Snapdeal.com and ebay.com. For each review, download the first reviews say 100 or 200 . Then Feature Extraction Algorithm is used to extract the product features and 
also separate the opinion targets and the opinion words based on the word alignment model. By identifying the opinion targets we can detect whether the sentence is positive or negative. By using topical relation, we can extract the relevant information or features only from a particular product from reviews.

\section{TABLE 1 Review Data Set}

\begin{tabular}{|l|l|}
\hline Data Items & Reviews \\
\hline Iron Box & $\begin{array}{l}\text { This is a very good and light weight press, } \\
\text { very good and attractive look. }\end{array}$ \\
\hline $\begin{array}{l}\text { Mobile } \\
\text { Phone }\end{array}$ & $\begin{array}{l}\text { High performance, Poor Battery life ,High } \\
\text { Resolution, etc. }\end{array}$ \\
\hline Trimmers & $\begin{array}{l}\text { Stunning Design, awesome product, It is } \\
\text { worth to money and good performance,etc. }\end{array}$ \\
\hline
\end{tabular}

We select the review datasets for Iron Box, mobile phone, Trimmers. Reviews are first segmented into sentence, next sentence are tokenized using standard NLP tool. By analyzing the datasets we can separate the opinion targets. Measuring the performance of the product based on the positive and negative opinion targets. In Fig 2, shows the performance evaluation of Digital camera, Mobile phone and laptops on various years. In Fig .3 Topical Relation, we can detect the performance of a particular features from particular products.

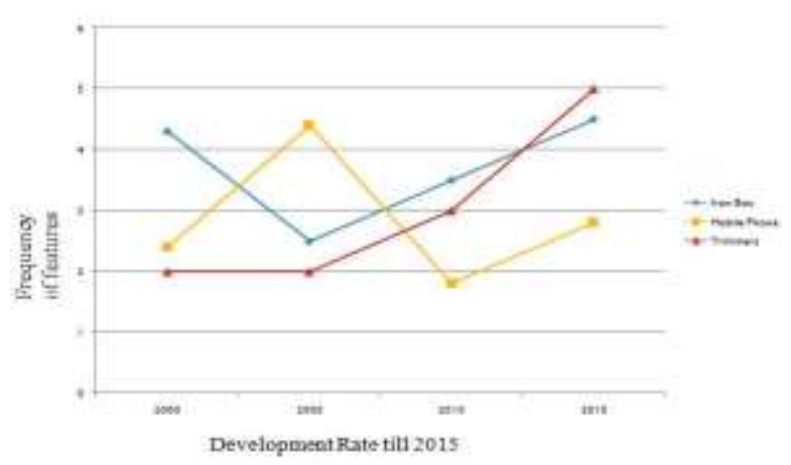

Figure .2 Performances of Products

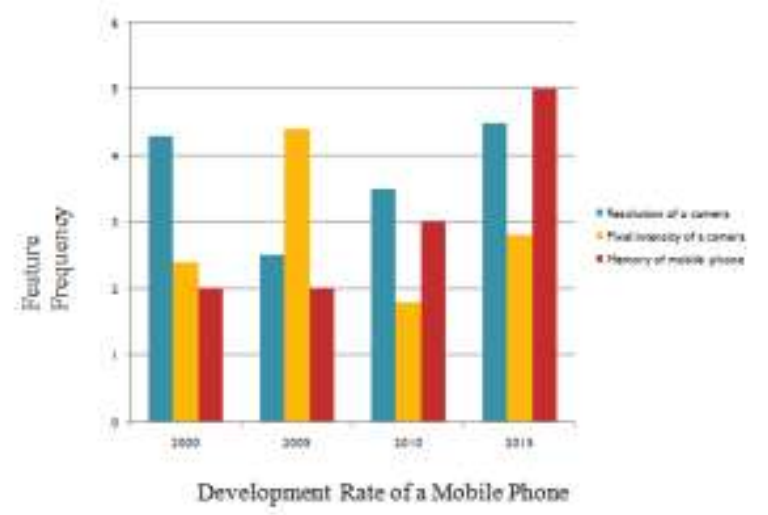

Figure.3 Product Improvement Based on Topical Relation

We can rank the products based on the customer reviews. By considering the Mobile phone, have positive and negative opinions. Fig:4 and Fig:5 showing the positive and negative ranking respectively.

There have lot of positive recommendations of the value of reviews for ecommerce, that the case doesn't really need to be made anymore, Fairly simply, user reviews increase conversions. They can eliminate any doubts possible customers may have about a product. In Fig: 4, shows that positive ranking of the mobile phone and Trimmer. Here $75 \%$ of reviewers say that the positive opinion about the memory of phone and $18 \%$ of pixel intensity and remaining reviews about the resolution of the phone. In case of Trimmer, 55\% of reviewers say that positive opinion about the attraction of trimmer and $33.3 \%$ of weight and $22 \%$ is about the battery life of a trimmer.
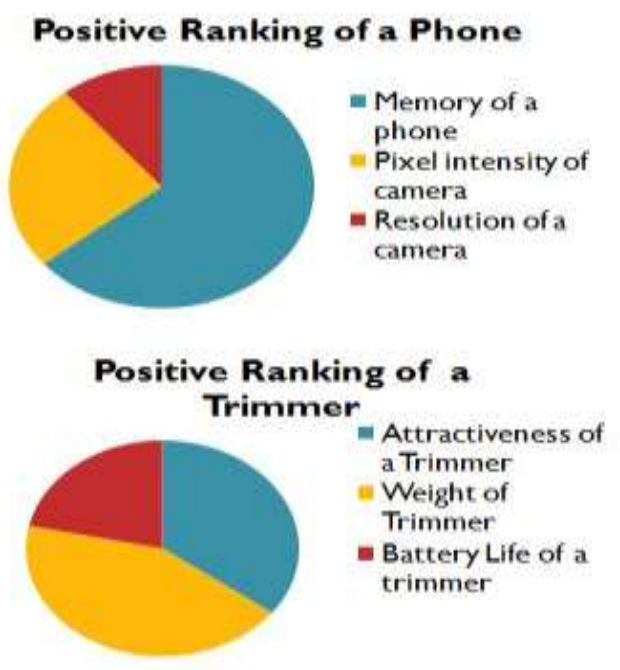

Figure 4. Positive Ranking

When purchasing items online, reading customer reviews is a suitable way to get a real account of other people's opinions of the product. Negative reviews that are set by a politeness-factor can actually help sell the item. In Fig:5, shows that negative ranking of the mobile phone. Here 25 of peoples say that the negative opinion about the memory and $76 \%$ of about pixel intensity and the remaining about the resolution of the phone. In case of Trimmer, $25 \%$ of reviewers say that negative opinion about the attraction of trimmer and $7 \%$ of weight and $68 \%$ is about the battery life of a trimmer. 
Negative Ranking of a Phone

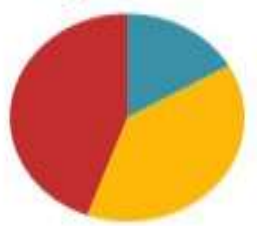

$$
\begin{aligned}
& \text { Memory of a } \\
& \text { phone } \\
& =\text { Pixel intensity of } \\
& \text { Camera } \\
& =\text { Resolution of a } \\
& \text { camera }
\end{aligned}
$$

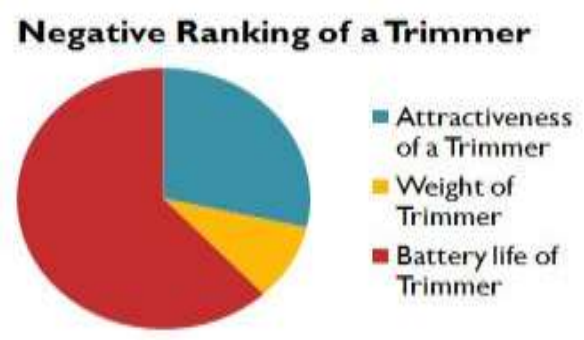

Figure.5 Negative Ranking of Mobile Phone

\section{CONCLUSIONS}

Opinions are the unique type of information which is different from facts. Joint information has spread all through the Web, particularly in areas connected to everyday life, like e commerce. Despite significant progress, however, opinion mining and sentiment analysis finding their own voice as new fields. This paper propose a Supervised word alignment model, which identifying the opinion relation. Rather than this paper focused on topical relation, in which to extract the relevant information or features only from a particular online reviews. Finally the items are ranked based on the frequency of positive and negative reviews.. We first classify the sentence as objective or subjective and then we analyze the adjectives or nouns thereby improving the performance. Compared to previous methods, our model captures opinion relation and feature extraction more precisely.

\section{REFERENCES}

[1] Kang Liu, Liheng Xu, and Jun Zhao, "Co-Extracting Opinion Targets and Opinion Words from Online Reviews Based on the Word Alignment Model", IEEE Trans. Knowledge and data Engineering, Vol. 27, No. 3, March 2015

[2] M. Hu and B. Liu, "Mining and summarizing customer reviews," in Proc. 10th ACM SIGKDD Int. Conf. Knowledge. Discovery Data Mining, Seattle, WA, USA, 2004

[3] A.Mukherjee and B. Liu, "Modeling review comments," in Proc.50th Annual. Meeting Assoc. comput. linguistics, Jeju, Korea, Jul.2012

[4] K. Liu, L. Xu, and J. Zhao, "Opinion target extraction using word based translation model," In Proc. Joint Conf. Empirical Methods Natural Lang. Process. Comput. Natural Lang. Learn., Jeju, Korea, Jul.2012

[5] Y. Wu, Q. Zhang, X. Huang, and L.Wu, "Phrase dependency parsing for Opinion mining," in Proc. Conf .Empirical methods Natural. Lang. Process, Singapore, 2009
[6] M. Hu and B. Liu, "Mining opinion features in customer reviews," in Proc.19th Nat. Conf. Artif. Intell., San Jose, CA, USA, 2004

[7] B. Wang and H. Wang, "Bootstrapping both product features and opinion words from Chinese customer reviews with cross inducing," in Proc.3rd Int. Joint Conf. Natural Lang. Process., Hyderabad, India, 2008

[8] Bo Pang, Lillian Lee, "Opinion Mining and Sentiment Analysis", Foundations and Trends in Information Retrieval Vol. 2, Nos. 1-2 (2008).

[9] L. Zhang, B. Liu, S. H. Lim, and E. O'Brien-Strain, "Extracting and ranking product features in opinion documents," in Proc. 23th Int.Conf. Comput. Linguistics, Beijing, China, 2010.

[10] J. Zhu, H. Wang, B. K. Tsou, and M. Zhu, "Multi-aspect opinion polling from textual reviews," in Proc. 18th ACM Conf. Inf. Knowl.Manage., Hong Kong, 2009, pp. 1799-1802.

[11] Z. Hai, K. Chang, J.-J. Kim, and C. C. Yang, "Identifying features in opinion mining via intrinsic and extrinsic domain relevance," IEEE Trans. Knowledge Data Eng., vol. 26, no. 3, p. 623-634, 2014.

[12] K. Liu, H. L. Xu, Y. Liu, and J. Zhao, “Opinion target extraction using partially-supervised word alignment model," in Proc. 23 $3^{\text {rd }}$ Int. Joint Conf. Artif. Intell., Beijing, China, 2013.

[13] A.M. Popescu and O. Etzioni, "Extracting product features and opinions from reviews," in Proc. Conf. Human Lang. Technol. Empirical Methods Natural Lang. Process., Vancouver, BC, Canada, 2005. 